\title{
Candida Biofilm Formation Assay on Essential Oil Coated Silicone Rubber
}

Gulcan Sahal $^{1, *}$, Herman J. Woerdenbag ${ }^{2}$, Wouter L. J. Hinrichs², Anita Visser ${ }^{3,4}$, Henny C. van der $\mathrm{Mei}^{5}$ and Isil Seyis Bilkay ${ }^{1}$

${ }^{1}$ Hacettepe University, Faculty of Sciences, Department of Biology (Biotechnology Division), Beytepe, Ankara, Turkey; ${ }^{2}$ University of Groningen, Department of Pharmaceutical Technology and Biopharmacy, Antonius Deusinglaan 1, Groningen, the Netherlands; ${ }^{3}$ University of Groningen and University Medical Center Groningen, Department of Oral and Maxillofacial Surgery and Maxillofacial Prosthodontics, Groningen, the Netherlands; ${ }^{4}$ University of Groningen and University Medical Center Groningen, Department of Geriatric Dentistry, Dental school, Center for Dentistry and Oral Hygiene, Groningen, the Netherlands; ${ }^{5}$ University of Groningen and University Medical Center Groningen, Department of Biomedical Engineering, Antonius Deusinglaan 1, Groningen, the Netherlands

*For correspondence: gulcanozbakir@gmail.com

[Abstract] Development of biofilm associated candidemia for patients with implanted biomaterials causes an urgency to develop antimicrobial and biofilm inhibitive coatings in the management of recalcitrant Candida infections. Recently, there is an increase in the number of patients with biofilm formation and resistance to antifungal therapy. Therefore, there is a growing interest to use essential oils as coating agents in order to prevent biomaterial-associated Candida infections. Often high costs, complicated and laborious technologies are used for both applying the coating and determination of the antibiofilm effects hampering a rapid screening of essential oils. In order to determine biofilm formation of Candida on essential oil coated surfaces easier, cheaper and faster, we developed an essential oil (lemongrass oil) coated surface (silicone-rubber) by using a hypromellose ointment/essential oil mixture. Furthermore, we modified the "crystal violet binding assay" to quantify the biofilm mass of Candida biofilm formed on the lemongrass oil coated silicone rubber surface. The essential oil coating and the biomass determination of biofilms on silicone rubber can be easily applied with simple and accessible equipment, and will therefore provide rapid information about whether or not a particular essential oil is antiseptic, also when it is used as a coating agent.

Keywords: Biofilm formation, Biomaterial infections, Candida, Candidemia, Essential oils, Essential oil coated surfaces

[Background] In recent years, the frequency of Candida infections caused by both nosocomial and opportunistic Candida strains has increased rapidly especially due to the growing number of transplant recipients, cancer patients and patients who receive immunosuppressive therapy (Sanguinetti et al., 2015). Apart from this, development of candidemia in patients with implanted biomaterials such as catheters (Simitsopoulou et al., 2014), dental implants (Li et al., 2012), voice prostheses (Talpaert et al., 2015) and prosthetic devices (Ramage et al., 2006) causes an urgency to develop preventive strategies such as antimicrobial coatings in the management of recalcitrant Candida infections (Ramage et al., 
2006). Since biofilm formation plays a pivotal role in the development of recalcitrant candidemia (Simitsopoulou et al., 2014), not only antimicrobial therapies, but also antibiofilm strategies are needed for the prevention of biomaterial-associated Candida infections. Recently, a growing increase in the incidence of candidemia has been associated with the increase in biofilm formation and resistance to antifungal therapy (Francisconi et al., 2020). Substances obtained from traditional medicinal plants, such as volatile essential oils have been regarded as attractive sources for new antimicrobials(Francisconi et al., 2020). Essential oils, originating from different parts of a variety of aromatic plants not only have antimicrobial but also have antibiofilm effects against various microorganisms (Akthar et al., 2014; Kim et al., 2016; Sahal et al., 2019 and 2020). Therefore, the antibiofilm effects of several essential oils against different Candida species have been studied for years (Almeida et al., 2016; Kryvtsova et al., 2019). Recently, essential oils have also been evaluated as agents that prevent biomaterial-associated Candida infections. In particular, there is a growing interest in the use of a lemongrass essential oil as a coating agent (Sahal et al., 2019 and 2020). Often high costs and complicated technologies are used for both applying the coating and determination of the antibiofilm effects. For example, Liakos et al. (2017) used electrospinning to apply a cellulose acetate based coating containing the essential oil and Anghel et al. (2012) used Confocal Laser Scanning Microscopy images to asses fungal biofilm architecture on $\mathrm{Fe}_{3} \mathrm{O}_{4} / \mathrm{C}_{18} /$ essential oil coated Provox ${ }^{\mathrm{TM}}$ voice prostheses. These techniques are often very expensive, laborious and require highly qualified equipment and personnel. Thus, they constrain an ease and rapid screening of essential oils. Therefore, we aimed to develop cheap, effective and easily applicable protocols to rapidly screen the anti-candida biofilm effects of various essential oils coated on silicone rubber.

In the first part, we describe the preparation of essential oil coated silicone rubber surface using a hypromellose ointment/essential oil mixture. In this part, hypromellose ointment was used as a vehicle because it mixes well with essential oils and because of its good adhesive properties to wetted surfaces. In the second part, we describe the determination of the biofilm mass of Candida biofilm on these surfaces using a modified crystal violet binding assay. Both protocols can be applied easily and rapidly with simple and accessible equipment.

\section{Materials and Reagents}

1. VST Silicone elastomer (low viscosity, translucent silicone) $(10 \times 10 \times 1.5 \mathrm{~mm}$ sheets) (Polymerization conditions: 5-bar pressure, $45{ }^{\circ} \mathrm{C}, 90 \mathrm{~min}$.) (Maxillofacial Silicone System, Technovent Ltd., South Wales, UK, catalog number: VST-50HD Shore A 38, cure time overnight).

2. Petri dish $(90 \mathrm{~mm} \times 17 \mathrm{~mm})$ (Isolab, catalog number: 08102061)

3. Erlenmayer Flask $(250 \mathrm{ml})$ (Isolab, catalog number: 027.01.250)

4. 6-Well plates (Biofil, catalog number: TCP 011006)

5. 24-Well plates (Biofil, catalog number: TCP 011024)

6. Centrifugal tube $50 \mathrm{ml}$ (Orange Scientific, catalog number: $4440100 \mathrm{~N}$ )

7. Candida strains (Clinical Candida isolates, previously characterized as highly biofilm forming 
and identified using the 18S Ribosomal RNA Gene Sequence Analysis; or biofilm-positive ATCC Candida strains such as Candida albicans (Robin) Berkhout (ATCC ${ }^{\circledR}$, catalog number: MYA$274^{\mathrm{TM}}$ )

8. Lemongrass essential oil (Merck, catalog number: 05521501)

9. Hypromellose ointment [Hypromellose $4000 \mathrm{mPa} . \mathrm{s} 20 \%(\mathrm{w} / \mathrm{w})$; Soft paraffin white $80 \%(\mathrm{w} / \mathrm{w})$ ], [FNA (Formularium der Nederlandse Apothekers), Dutch Pharmacists' Formulary, 2013], (Fagron, catalog number: 102064)

10. Crystal Violet Powder (Merck, catalog number: C0775-100G)

11. Brain Heart Infusion (BHI) Powder (Merck, catalog number: M110493.0500)

12. Agar-agar (Merck, catalog number: M101613.0500)

13. Ethanol $96 \%$ (v/v) (Isolab, catalog number: 9200262500)

14. Extran MA 02 (Merck, catalog number: 107553)

15. $\mathrm{K}_{2} \mathrm{HPO}_{4}$ (Merck, catalog number: 7758-11-4)

16. $\mathrm{KH}_{2} \mathrm{PO}_{4}$ (Merck, catalog number: 7778-77-0)

17. Distilled Water

18. Brain Heart Infusion Broth (see Recipes)

19. Brain Heart Infusion Agar (see Recipes)

20. $1 \%$ Crystal Violet Solution (see Recipes)

21. $10 \mathrm{mM}$ Potassium phosphate buffer (see Recipes)

\section{Equipment}

1. Bunsen burner

2. Porcelain mortar [Outside diameter $10.5 \mathrm{~cm}$, inner diameter $9.0 \mathrm{~cm}$, inner depth $4.5 \mathrm{~cm}$. Content $100 \mathrm{ml}$. Glazed to grinding surface, grinding surface unglazed and porcelain pestle (Length 11 $\mathrm{cm}$, unglazed on grinding surface, further glazed)] (Haldenwanger, Berlin)

3. Forceps

4. Spectrophotometer (UV-Visible Spectrophotometer; Shimadzu, model: UV-1700, catalog number: 206-98366A)

5. Centrifuge (Eppendorf, model: 5810R, with an Eppendorf Swing-bucket rotor A-4-62, catalog number: 5810000327)

6. $\mathrm{pH}$ Meter (Consort ${ }^{\mathrm{TM}} \mathrm{C} 3010$ Benchtop Multiparameter Analyzer) (Fisher Scientific, catalog number: 11772009)

7. Thoma Counting Chamber (Neubauer, catalog number: C964110)

8. Autoclave (Priorclave, Catalog number: 12758935)

9. Light Microscope (LEICA, model: DM500)

10. Incubator (Static) (Dedeoglu, catalog number: MMF-KM-068)

\section{Software}


1. MINITAB (Version 18)

2. ImageJ (Version 1.49)

\section{Procedure}

A. Preparation of essential oil coated silicone rubber surfaces by using hypromellose ointment (see also Figure 1)

1. Mix hypromellose ointment with a particular essential oil (w/w) under aseptic conditions using a sterile porcelain mortar and pestle.

2. Place a layer of essential oil/hypromellose ointment mixture $(50 \mathrm{mg})(\mathrm{w} / \mathrm{w})$ in a sterile Petri dish under sterile conditions by using a Bunsen burner.

3. Clean silicone rubber surface sheets by rinsing them with $2 \%$ Extran MA02 in water followed by tap water, $70 \%$ ethanol solution ( $\mathrm{v} / \mathrm{v}$ ) and sterile demineralized water.

4. Place the sterile silicone rubber surface sheet $(10 \times 10 \mathrm{~mm})$ on top of a layer of essential oil/hypromellose ointment mixture (w/w).

5. Apply a gentle pressure onto the silicone rubber surface sheet to let the essential oil/hypromellose ointment mixture stick onto the material.

6. Take essential oil coated silicone rubber surface sheet gently out of the Petri dish by using sterile forceps and place it in a well of a sterile 6-Well plate for the further analysis.

7. Place the essential oil coated silicone rubber surface sheet vertical on a sterile Petri dish and put them under a light microscope $(\times 10)$ in order to capture an image that contains thicknesses of both the silicone rubber sheet and the coating.

8. Set the thickness of the silicone rubber sheet as $1.5 \mathrm{~mm}$ and estimate the thickness of the essential oil/hypromellose ointment coatings on the silicone rubber surfaces by using Image J Software.
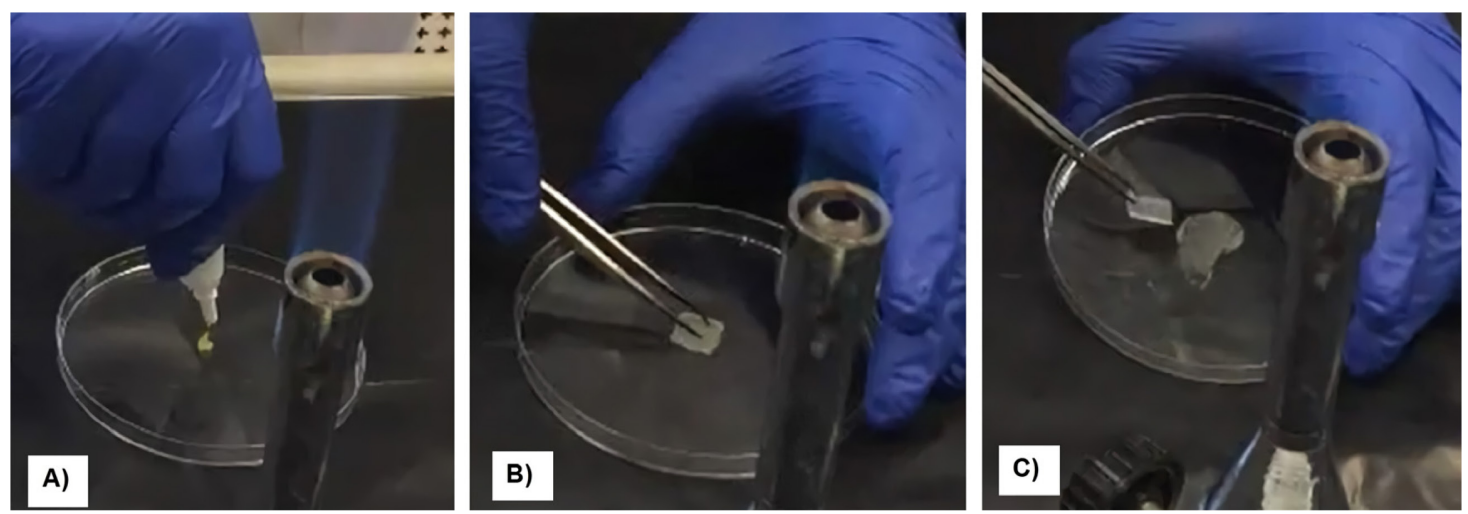

Figure 1. Preparation of essential oil coated silicone rubber by using essential oil/hypromellose ointment mixture. A. Placement of essential oil/hypromellose ointment mixture in a sterile Petri dish. B. Placement of a sterile biomaterial surface sheet on top of a layer of essential oil/hypromellose ointment mixture and application of a gentle pressure onto 
the biomaterial surface sheet to let the essential oil/hypromellose ointment mixture stick onto the material. C. Taking essential oil coated surface sheet gently out of the Petri dish.

B. Determination of Candida biofilm formation on essential oil coated surfaces (see also Figure 2)

1. Inoculate Candida strains from stock culture (grown in $\mathrm{BH}$ with glycerol) into Brain Heart Infusion (BHI) agar by a streak plating technique and incubate them for $24 \mathrm{~h}$ at $37^{\circ} \mathrm{C}$ under aerobic conditions.

2. Inoculate single colonies of Candida strains for pre-culture preparations into $10 \mathrm{ml} \mathrm{BHI}$ broth and incubate overnight at $37^{\circ} \mathrm{C}$ without shaking.

3. Inoculate $10 \mathrm{ml}$ preculture into the $200 \mathrm{ml} \mathrm{BHI}$ broth and grow it for $24 \mathrm{~h}$ at $37^{\circ} \mathrm{C}$ without shaking to obtain main culture.

4. Harvest Candida cells by centrifugation at $3,220 \times g$ for 10 min at $5{ }^{\circ} \mathrm{C}$ and wash 3 times with $30 \mathrm{ml} 10 \mathrm{mM}$ potassium phosphate buffer ( $\mathrm{pH} 7.0)$.

5. Count Candida cells that exist in 8 different medium squares of a Thoma Counting Chamber under a light microscope with $40 \times$ objective. Calculate the average of the cells for a single medium square and calculate the Candida cell density considering the volume of a single medium square of the Thoma Counting Chamber. Adjust cell density to $3 \times 10^{6} \mathrm{cells} / \mathrm{ml}$ in BHI broth.

6. Place the essential oil coated silicone rubber surfaces, silicone rubber surfaces with only hypromellose ointment and the silicone rubber surfaces without any coating (positive controls) into the wells of a 24 -well plate.

7. Add $2 \mathrm{ml}$ of Candida suspensions to the wells of 24 -well plates that include the different silicone rubber surfaces and incubate them for 7 days at $37^{\circ} \mathrm{C}$ under aerobic conditions without shaking.

8. After 7 days of incubation, wash gently three times the materials harboring biofilm with $10 \mathrm{mM}$ potassium phosphate buffer $(\mathrm{pH} 7)$ and stain them with a $1 \%(\mathrm{w} / \mathrm{v})$ solution of crystal violet in sterile distilled water for $20 \mathrm{~min}$ at $25^{\circ} \mathrm{C}$.

9. Subsequently, gently rinse the materials with $10 \mathrm{mM}$ potassium phosphate buffer $(\mathrm{pH} 7.0)$ again until no crystal violet residue left in the washing solution.

10. Dissolve crystal violet bound to the biofilm on the different tested surfaces in $2 \mathrm{ml}$ ethanol (96\%; $\mathrm{v} / \mathrm{v}$ ) for $20 \mathrm{~min}$ (O'Toole, 2011).

11. Measure the absorbance of dissolved crystal violet at $560 \mathrm{~nm}$ using a spectrophotometer (O'Toole, 2011).
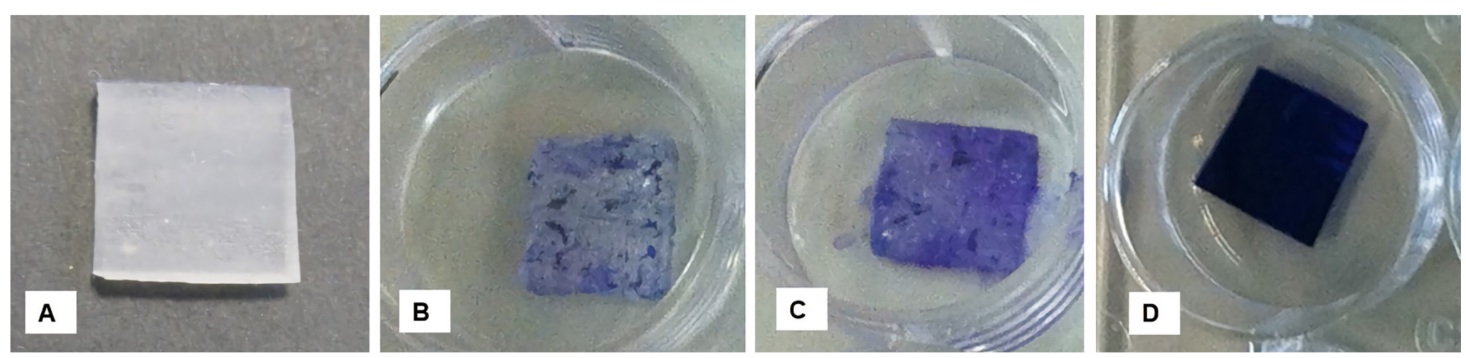
Figure 2. Biofilm formation of Candida. Images of A) silicone rubber surface B) Biofilm formation on $8 \%$ Lemongrass oil coated silicone rubber surface $\mathrm{C}$ ) Biofilm formation on $0 \%$ Lemongrass oil coated silicone rubber surface (hypromellose ointment alone) D) Biofilm formation on silicone rubber without coating (positive control).

\section{Data analysis}

1. Set the absorbance value of the silicone rubber surface without any coating and microorganism, but following the same procedure as the biofilm growth and crystal violet staining, as a negative control. Subtract this value from the absorbance values of the surfaces with biofilms.

2. Evaluate biofilm formation on the silicone rubber surface without coating as a positive control and set it as $100 \%$. Set silicone rubber surface coated with hypromellose ointment without any essential oil as $0 \%$ essential oil coated surface.

3. Measure the absorbance values of biofilm formations of treatments and calculate decrease (\%) in biofilm formation of treatments relative to the positive control, using the equation:

$\%$ Decrease $=[($ Absorbance Positive Control $(560 \mathrm{~nm})-$ Absorbance Treatment $(560$ $\mathrm{nm})) /$ Absorbance Positive Control $(560 \mathrm{~nm})] \times 100 \%$

4. Perform statistical analysis using MINITAB 18 software.

5. Apply Anderson-Darling test to determine if biofilm formation and biofilm inhibition data were normally distributed. Following this, apply Levene's test to assess homogeneity of variances.

6. In case the results are found to be normally distributed, apply two factor experimental design and do pairwise comparisons by the Tukey test. In case the results are not found as normally distributed, use non-parametric tests (Kruskal-Wallis and Mann-Whitney U tests) to evaluate the results.

\section{Notes}

1. Determination of biofilm formation of Candida on essential oil coated silicone rubber surfaces by modified crystal violet binding assay need to be performed at least in triplicate, including controls.

2. When the absorbance is too high, dilute the dissolved crystal violet and multiply the absorbance value by the dilution factor.

\section{Recipes}

1. Brain Heart Infusion Broth $(1 \mathrm{~L})$

Dissolve $37 \mathrm{~g} \mathrm{BHI}$ powder in $1 \mathrm{~L}$ distilled water and sterilize the medium at $121^{\circ} \mathrm{C}$ for $15 \mathrm{~min}$ 
using an autoclave.

2. Brain Heart Infusion Agar (1 L)

Dissolve $37 \mathrm{~g} \mathrm{BHI}$ powder and $30 \mathrm{~g}$ Agar-Agar powder in $1 \mathrm{~L}$ distilled water and place the media into sterile Petri dishes after sterilization at $121^{\circ} \mathrm{C}$ for 15 min using an autoclave.

3. $1 \%$ Crystal Violet Solution (w/v)

Dissolve $5 \mathrm{~g}$ crystal violet in $500 \mathrm{ml}$ distilled water and incubate the crystal violet solution (1\%, $\mathrm{w} / \mathrm{v}$ ) in shaking incubator for $2 \mathrm{~h}$ at $150 \mathrm{rpm}$, at room temperature.

4. $10 \mathrm{mM}$ Potassium Phosphate Buffer ( $\mathrm{pH} 7.0)(1 \mathrm{~L})$

a. Dissolve $4.35 \mathrm{~g} \mathrm{~K}_{2} \mathrm{HPO}_{4}$ and $3.4 \mathrm{~g} \mathrm{KH}_{2} \mathrm{PO}_{4}$ in $100 \mathrm{ml}$ distilled water to obtain stock $0.5 \mathrm{M}$ Potassium Phosphate Buffer.

b. Dilute $0.5 \mathrm{M}$ potassium phosphate buffer to $10 \mathrm{mM}$ potassium phosphate buffer.

c. Adjust the $\mathrm{pH}$ of the buffer to 7.0 by using $\mathrm{pH}$ meter and sterilize the buffer at $121^{\circ} \mathrm{C}$ for 15 min using an autoclave.

\section{Acknowledgments}

This study was supported by funding received from Scientific Research Projects Coordination Unit (grant number: FDK-2016-10821) of Hacettepe University, Ankara, Turkey. This protocol was derived from Sahal et al. (2020).

\section{Competing interests}

The authors declare no competing financial interests.

\section{$\underline{\text { References }}$}

1. Akthar, M. S., Degaga, B. and Azam, T. (2014). Antimicrobial activity of essential oils extracted from medicinal plants against the pathogenic microorganisms: A review. Biol Sci Pharm Res 2(1): 1-7.

2. Almeida, Lde, F., Paula, J. F., Almeida, R. V., Williams, D. W., Hebling, J. and Cavalcanti, Y. W. (2016). Efficacy of citronella and cinnamon essential oils on Candida albicans biofilms. Acta Odontol Scand 74(5): 393-398.

3. Anghel, I., Grumezescu, V., Andronescu E., Anghel G. A. Grumezescu, A. M., Mihaiescu, D. E. and Chifiriuc M. C. (2012). Protective effect of magnetite nanoparticle/Salvia officinalis essential oil hybrid nanobiosystem against fungal colonization on the Provox $®$ voice section prosthesis. Dig J Nanomater Biostructures 7(3): 1205-1212.

4. Francisconi, R. S., Huacho, P. M. M., Tonon, C. C., Bordini, E. A. F., Correia, M. F., Sardi, J. C. O. and Spolidorio, D. M. P. (2020). Antibiofilm efficacy of tea tree oil and of its main component terpinen-4-ol against Candida albicans. Braz Oral Res 34: e050. 
5. Kim, Y. G., Lee, J. H., Gwon, G., Kim, S. I., Park, J. G. and Lee, J. (2016). Essential Oils and Eugenols Inhibit Biofilm Formation and the Virulence of Escherichia coli 0157:H7. Sci Rep 6: 36377.

6. Kryvtsova, M. V., Salamon, I., Koscova, J., Bucko, D. and Spivak, M. (2019). Antimicrobial, antibiofilm and biochemichal properties of Thymus vulgaris essential oil against clinical isolates of opportunistic infections. Biosyst Divers 27(3): 270-275.

7. Li, J., Hirota, K., Goto, T., Yumoto, H., Miyake, Y. and Ichikawa, T. (2012). Biofilm formation of Candida albicans on implant overdenture materials and its removal. J Dent 40(8): 686-692.

8. Liakos, I. L., Holban, A. M., Carzino, R., Lauciello, S. and Grumezescu, A. M. (2017). Electrospun Fiber Pads of Cellulose Acetate and Essential Oils with Antimicrobial Activity. Nanomaterials (Basel) 7(4): 84.

9. O'Toole, G. A. (2011). Microtiter dish biofilm formation assay. J Vis Exp 47: 2437.

10. Ramage, G., Martinez, J. P. and Lopez-Ribot, J. L. (2006). Candida biofilms on implanted biomaterials: a clinically significant problem. FEMS Yeast Res 6(7): 979-986.

11. Sahal, G., Woerdenbag, H. J., Hinrichs, W. L. J., Visser, A., Tepper, P. G., Quax, W. J., van der Mei, H. C. and Bilkay, I. S. (2020). Antifungal and biofilm inhibitory effect of Cymbopogon citratus (lemongrass) essential oil on biofilm forming by Candida tropicalis isolates; an in vitro study. $J$ Ethnopharmacol 246: 112188.

12. Sahal, G., Nasseri, B., Ebrahimi, A. and Bilkay, I. S. (2019). Electrospun essential oilpolycaprolactone nanofibers as antibiofilm surfaces against clinical Candida tropicalis isolates. Biotechnol Lett 41(4-5): 511-522.

13. Sanguinetti, M., Posteraro, B. and Lass-Florl, C. (2015). Antifungal drug resistance among Candida species: mechanisms and clinical impact. Mycoses 58 Suppl 2: 2-13.

14. Simitsopoulou, M., Kyrpitzi, D., Velegraki, A., Walsh, T. J. and Roilides, E. (2014). Caspofungin at catheter lock concentrations eradicates mature biofilms of Candida lusitaniae and Candida guilliermondii. Antimicrob Agents Chemother 58(8): 4953-4956.

15. Talpaert, M. J., Balfour, A., Stevens, S., Baker, M., Muhlschlegel, F. A. and Gourlay, C. W. (2015). Candida biofilm formation on voice prostheses. J Med Microbiol 64(Pt 3): 199-208. 